\title{
The Combined Performance of ADC, CSF CXC Chemokine Ligand 13, and CSF Interleukin 10 in the Diagnosis of Central Nervous System Lymphoma
}

\author{
M.C. Mabray, R.F. Barajas, J.E. Villanueva-Meyer, C.A. Zhang, F.E. Valles, J.L. Rubenstein, and S. Cha
}

\begin{abstract}
BACKGROUND AND PURPOSE: CXC chemokine ligand 13 and interleukin 10 have emerged as CSF biomarkers for the diagnosis of CNS lymphoma. Our hypothesis is that the combined use of ADC, CXC chemokine ligand 13, and interleukin 10 will result in increased diagnostic performance compared with the use of ADC values alone.
\end{abstract}

MATERIALS AND METHODS: Eighty-seven patients were included in this study, including 43 with CNS lymphoma and 44 without CNS lymphoma (21 metastases, 14 high-grade gliomas, 9 tumefactive demyelinating lesions) who had undergone CSF proteomic analysis and had a new enhancing mass on brain MR imaging. Average ADC was derived by contouring the contrast-enhancing tumor volume. Group means were compared via $t$ tests for average ADC, CXC chemokine ligand 13, and interleukin 10. Receiver operating characteristic analysis was performed for each individual variable. Multiple-variable logistic regression with receiver operating characteristic analysis was performed, and the multiple-variable receiver operating characteristic was compared with single-variable receiver operating characteristics.

RESULTS: The average ADC was lower and CSF CXC chemokine ligand 13 and interleukin 10 values were higher in CNS lymphoma ( $P<.001)$. Areas under the curve ranged from 0.739 to 0.832 for single-variable ROC. Multiple-variable logistic regression yielded statistically significant individual effects for all 3 variables in a combined model. Multiple-variable receiver operating characteristics (area under the curve, 0.928 ) demonstrated statistically significantly superior diagnostic performance compared with the use of single variables alone.

CONCLUSIONS: The combined use of ADC, CSF CXC chemokine ligand 13, and interleukin 10 results in increased diagnostic performance for the diagnosis of CNS lymphoma. This finding highlights the importance of CSF analysis when the diagnosis of CNS lymphoma is considered on the basis of MR imaging.

ABBREVIATIONS: $A$ DCavg = average ADC; CNSL = central nervous system lymphoma; $C X C L-13=$ CXC chemokine ligand $13 ; \mathrm{lL}-10=$ interleukin 10; $R O C=$ receiver operating characteristic; $A \cup C=$ area under the curve

C entral nervous system lymphoma (CNSL) may be primary CNSL when isolated to the central nervous system or secondary CNSL in the setting of systemic lymphoma. ${ }^{1-3}$ Patients who

Received February 26, 2015; accepted after revision May 12.

From the Departments of Radiology and Biomedical Imaging (M.C.M., R.F.B., J.E.V.-M., C.A.Z., F.E.V., S.C.), Medicine Division of Hematology/Oncology (J.L.R.), Epidemiology and Biostatistics (C.A.Z.), and Neurological Surgery (S.C.), University of California San Francisco, San Francisco, California.

M.C.M. and R.F.B. contributed equally to this study and are considered co-first authors. J.L.R. and S.C. contributed equally to this study and are considered co-senior authors.

M.C.M. and R.F.B. were supported by a National Institutes of Health T32 training grant (5T32EB001631-10).

Paper previously presented at: Annual Meeting of the Radiological Society of North America, November 30 to December 5, 2014; Chicago, Illinois.

Please address correspondence to Marc C. Mabray, MD, Radiology and Biomedical Imaging, 505 Parnassus Ave, M391, University of California, San Francisco, San Francisco, CA 94143; e-mail: marc.mabray@ucsf.edu, marccm7@gmail.com

-- Indicates open access to non-subscribers at www.ajnr.org

http://dx.doi.org/10.3174/ajnr.A4450 are immunocompromised are at an increased risk of developing CNSL (primary or secondary); however, the rates of primary CNSL are increasing among immunocompetent patients. ${ }^{4-8}$ CNSL now accounts for approximately $1 \%-5 \%$ of all brain tumors and thus should be considered in the differential diagnosis of a patient with a new brain mass lesion., ${ }^{4,6,7}$

Arriving at a consistently accurate preoperative diagnosis for a patient with a brain mass lesion encountered on MR imaging remains an overall difficult task. ${ }^{9}$ There is considerable overlap in the clinical presentation and appearance of brain mass lesions on MR imaging, including CNSL, and there is much research into using advanced imaging techniques to arrive at a diagnosis. ${ }^{9-13}$ Ultimately, most patients with a newly encountered brain mass lesion will undergo stereotactic brain biopsy to arrive at a diagnosis, an invasive procedure with a rate of diagnostic failure that may be as high as $35 \% .{ }^{14,15}$ The diagnosis of CNSL may be further complicated by its response to glucocorticoids, which may com- 


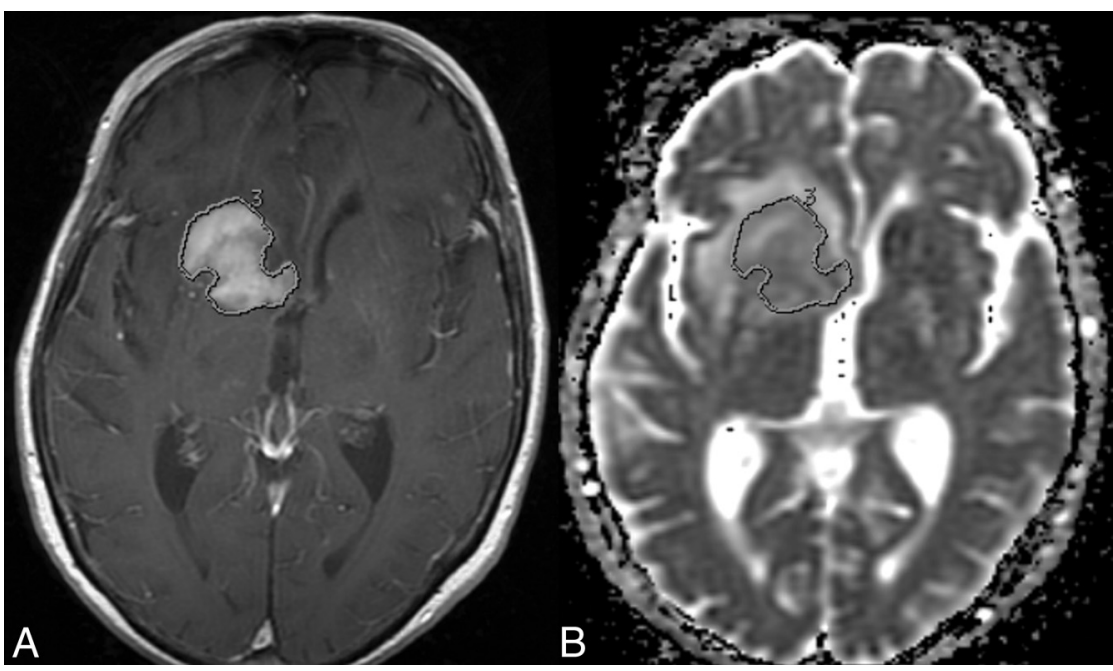

FIG 1. An axial postgadolinium T-weighted MR image $(A)$ and a coregistered axial ADC map (B) demonstrate manual contouring of the contrast-enhancing CNSL on this section, which allows the measurement of ADCavg. This is performed on every section of the contrast-enhancing tumor. undergone CSF sampling from 2003 to 2011 at our institution as part of a multiinstitutional study. ${ }^{22,24}$ We cross-referenced this data base with MR imaging. Inclusion criteria for our study were CSF sampling with CXCL-13 and IL-10 and a preoperative brain MR imaging showing an enhancing mass lesion. Exclusion criteria were age younger than 12 years, traumatic CSF collection, or therapeutic intervention within 3 weeks. Eighty-seven patients were included in this study, including 43 with CNSL ( 38 primary CNSL, 5 secondary CNSL) and 44 without CNSL (21 metastases, 14 high-grade gliomas, 9 tumefactive demyelinating lesions). All diagnoses were pathologically confirmed following surgical biopsy. None of the patients were HIV-positive. The medical record was retrospectively reviewed to determine whether patients received plicate diagnosis by both MR imaging and stereotactic biopsy. ${ }^{16,17}$ tk;4Ideally, an accurate diagnosis of a new brain mass lesion could be offered before brain biopsy to facilitate treatment planning and surgical triage, and potentially even obviate biopsy in some cases.

Multiple MR imaging features have been reported useful for distinguishing CNSL from the more common high-grade glioma, including relatively low ADC values on DWI, relatively low cerebral blood volume and high permeability on MR perfusion, lipid peaks and high choline-to-creatinine ratios on MR spectroscopy, and the absence of foci of susceptibility on high-resolution susceptibility imaging. ${ }^{10-13,18,19}$ ADC derived from DWI is of particular interest because this sequence is routinely performed on all brain MRI. Low ADC values in CNSL are related to high cellularity, which theoretically interferes with the diffusion of water within the tumor. ${ }^{20,21}$ These MR imaging findings may be helpful but are also present in numerous other conditions and are ultimately nonspecific.

CXC chemokine ligand 13 (CXCL-13), a mediator of B-cell migration, and interleukin 10 (IL-10), an anti-inflammatory cytokine, are produced by lymphocytes in CNSL, can be detected in the CSF, and have emerged as CSF biomarkers for the diagnosis of CNSL. ${ }^{22-25}$ These CSF biomarkers should be considered in the evaluation of a brain mass detected on MR imaging. The aim of our study was to investigate the performance of the combined use of ADC derived from the preoperative clinical MR imaging and CSF CXCL-13 and IL-10 concentrations for the diagnosis of CNSL. Our hypothesis is that the combined use of ADC, CSF CXCL-13, and CSF IL-10 will result in increased diagnostic performance compared with the diagnostic performance of ADC values alone.

\section{MATERIALS AND METHODS \\ Study Subjects}

Eighty-seven patients were included in this cohort study compliant with the institutional review board and Health Insurance Portability and Accountability Act. We performed a retrospective review of a prospectively built cohort data base of patients who had corticosteroids before CSF sampling.

\section{CSF Proteomic Analysis}

These methods have been previously described. ${ }^{22,24}$ Briefly, CSF samples were frozen within 2 hours of collection and stored at $-70^{\circ} \mathrm{C}$ until analysis, at which point they were thawed and CSF concentrations of CXCL-13 and IL-10 were determined in duplicate by using an enzyme-linked immunoabsorbent assay. All CSF CXCL-13 and IL-10 concentrations are reported in picograms per milliliter.

\section{MR Imaging and ADC Measurements}

Standard clinical MR imaging was performed at $1.5 \mathrm{~T}(n=68)$ or $3 \mathrm{~T}$ $(n=19)$. Axial DWI (TR/TE, 10,000/99 ms; section thickness/intersection gap, 5/0 mm; matrix size, $256 \times 256$; FOV, $24 \mathrm{~cm} \times 24 \mathrm{~cm} ; 3$ orthogonal diffusion gradient directions; b-values, 0 and 1000 $\mathrm{s} / \mathrm{mm}^{2}$ ) was performed in the transverse plane covering the whole brain. Contrast-enhanced 3D spoiled gradient-recalled T1-weighted imaging (TR/TE, 34/8 ms; section thickness/intersection gap, 1.5/0 $\mathrm{mm}$ ) was performed in the axial plane. Slight variations in the scanning protocol were allowed as changes were made in the departmental protocol with time, as long as the patients had diffusion-weighted imaging performed at $b=0$ and $1000 \mathrm{~s} / \mathrm{mm}^{2}$.

All MR images were initially reviewed on the clinical PACS. We performed additional image processing and analysis in a blinded fashion off-line from the clinical PACS workstation using the FuncTool application (Version 9.4.05a; GE Healthcare, Milwaukee, Wisconsin) of an Advantage Workstation (Version 4.5; GE Healthcare). ADC maps were constructed from the diffusionweighted images and coregistered to the postcontrast T1weighted images. Each lesion was manually segmented on each section of the ADC map by contouring the contrast-enhancing lesion on the postcontrast T1-weighted images (Fig 1) by using established methods. ${ }^{21,26}$ Manual adjustments were made on the ADC map if there was any misregistration. Average ADC (ADCavg) was calculated for each lesion volume in units of $10^{-6}$ $\mathrm{mm}^{2} / \mathrm{s}$ (all ADC values in this article are reported in units of $10^{-6}$ $\mathrm{mm}^{2} / \mathrm{s}$ ). All ROIs were performed by a neuroradiology trainee 
(R.F.B.) and secondarily approved by an attending neuroradiologist (S.C.) certified by the American Board of Radiology with a Certificate of Added Qualification in neuroradiology.

\section{Statistical Analysis}

Statistical analysis and line art production were performed by using MedCalc for Windows, Version 14.8.1 (MedCalc Software, Mariakerke, Belgium) and the R statistical computing software

Table 1: Patient characteristics ${ }^{\text {a }}$

\begin{tabular}{lccc}
\hline & $\begin{array}{c}\text { Overall } \\
(\boldsymbol{n}=\mathbf{8 7})\end{array}$ & $\begin{array}{c}\text { CNSL } \\
(\boldsymbol{n}=\mathbf{4 3})\end{array}$ & $\begin{array}{c}\text { Non-CNSL } \\
(\boldsymbol{n}=\mathbf{4 4})\end{array}$ \\
\hline Mean age (yr) (SD) & $56.03(16.97)$ & $61.84(15.20)$ & $49.95(16.57)$ \\
Age range (yr) & $15-85$ & $24-84$ & $15-85$ \\
Male/female ratio & $43: 44$ & $20: 23$ & $23: 21$ \\
\hline
\end{tabular}

${ }^{a}$ The patients with CNSL were statistically significantly older than the patients without CNSL $(P=.001)$.

Table 2: Variables for the CNSL and non-CNSL groups with $P$ values $^{a}$

\begin{tabular}{cccc}
\hline & \multicolumn{3}{c}{ Variable Means by Patient Group } \\
\cline { 2 - 4 } Factor & CNSL $(\boldsymbol{n}=43)$ & Non-CNSL $(\boldsymbol{n}=44)$ & $\boldsymbol{P}$ Value \\
\hline ADCavg & 864.81 & 1071.65 & $<.001$ \\
$95 \% \mathrm{Cl}$ & $(832.50-897.13)$ & $(981.23-1162.04)$ & \\
CXCL-13 & 2960.49 & 72.54 & $<.001$ \\
$95 \% \mathrm{Cl}$ & $(1124.96-4796.01)$ & $(9.02-136.05)$ & \\
$\mathrm{IL}-10$ & 557.48 & 5.93 & $<.001$ \\
$95 \% \mathrm{Cl}$ & $(167.49-947.47)$ & $(3.42-8.43)$ & \\
\hline
\end{tabular}

a The patients with CNSL had lower ADC values and higher CSF CXCL-13 and IL-10 values than those without CNSL. (http://www.r-project.org). The mean and SD of age were calculated for all patients and for the 2 patient groups, which were compared with a Welch 2-tailed $t$ test. Corticosteroid administration was compared between the 2 patient groups with a Fisher exact test. Mean and 95\% confidence intervals for the CNSL and non-CNSL groups were calculated for ADCavg, CXCL-13, and IL-10, and means were compared with Welch 2-tailed $t$ tests. Single-variable receiver operating characteristic (ROC) analysis was then conducted for each variable for the diagnosis of CNSL. Multiple-variable logistic regression with ROC was then performed for the identification of CNSL by using the predictive variables ADCavg, CXCL-13, and IL-10. Optimized sensitivities and specificities were identified by using the maximum Youden J Index (maximum vertical distance to the null hypothesis AUC $=0.5$ line or sensitivity [1-specificity]). Additionally, thresholds required for $95 \%$ specificity were calculated along with the sensitivity at this threshold. Pair-wise comparisons of the multiple-variable ROC curve AUCs were made to the single-variable ROC curves by using the ROC compare function of MedCalc, which uses the method of Delong et al, ${ }^{27}$ accounting for correlated variables. A two-tailed $P<.05$ was considered statistically significant.

\section{RESULTS}

\section{Patient Characteristics}

Patient characteristics are reported in Table 1. The CNSL group (mean age, 61.84 years) was statistically significantly older than the non-CNSL group (mean age, 49.95 years) $(P=.001)$. Corticosteroid administration information before CSF sampling was

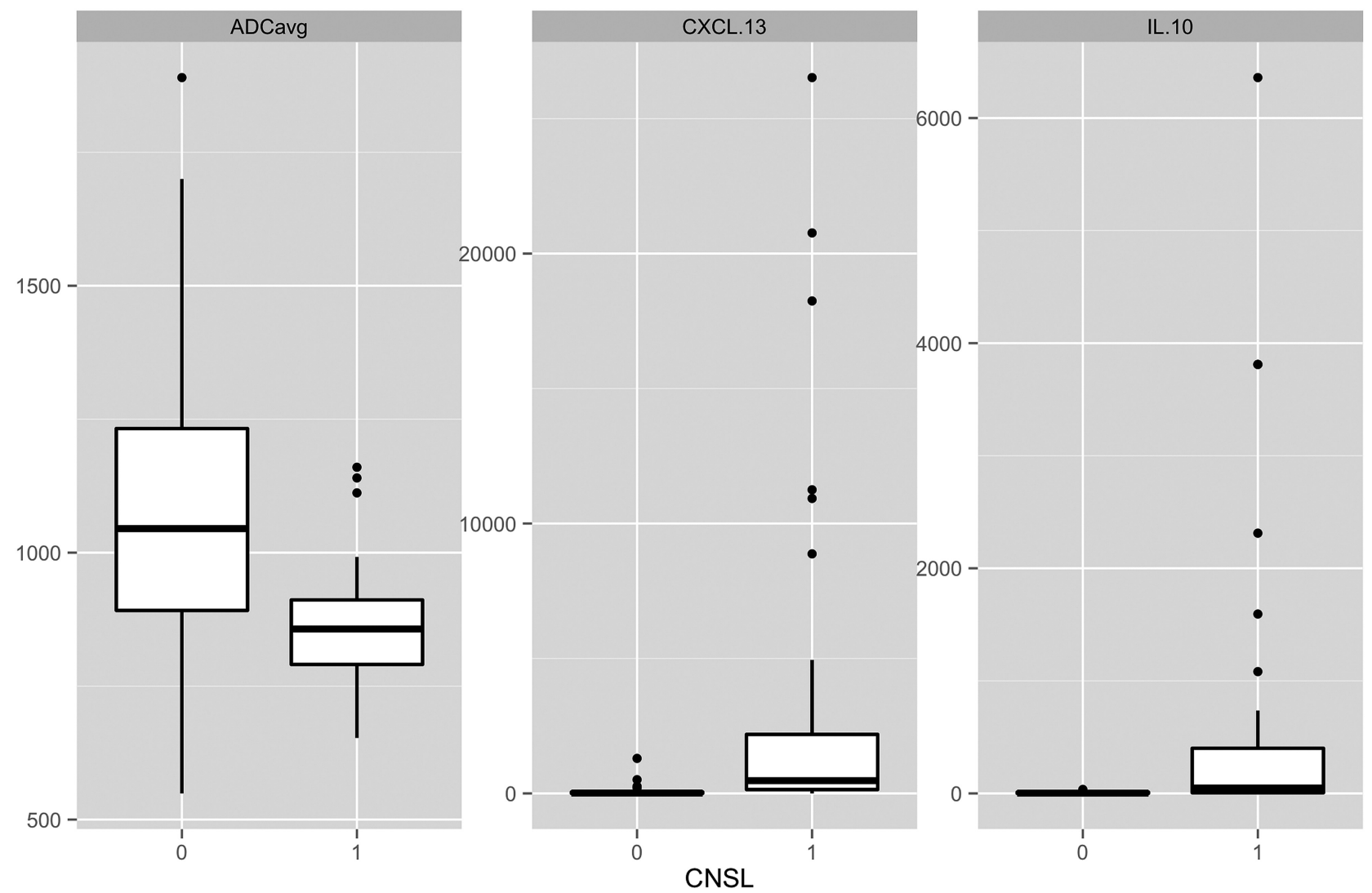

FIG 2. Boxplots of ADCavg, CSF CXCL-13, and CSF IL-10. ADCavg is statistically significantly lower, and CSF CXCL-13 and IL-10 are statistically significantly higher in patients with CNSL $(P<.001)$. 
Table 3: Results of individual and multiple-variable ROCs for the diagnosis of CNSL ${ }^{\mathrm{a}}$

\begin{tabular}{|c|c|c|c|c|c|}
\hline \multirow[b]{2}{*}{ Factor and $P$ Value } & \multicolumn{5}{|c|}{ ROC Results with Optimized Thresholds } \\
\hline & $\operatorname{AUC}(95 \% \mathrm{CI})$ & $\begin{array}{l}\text { Maximum Youden } \\
\text { Index J }\end{array}$ & $\begin{array}{c}\text { Threshold } \\
\text { Sensitivity/Specificity }\end{array}$ & $\begin{array}{l}\text { Threshold } \pm \text { Likelihood } \\
\text { Ratios }\end{array}$ & $\begin{array}{l}\text { Threshold } \\
\text { PPV/NPV }\end{array}$ \\
\hline ADCavg $(P<.001)$ & $0.739(0.634-0.827)$ & 0.521 at $\leq 971$ & $90.70 / 61.36$ & $2.35 / 0.15$ & $69.64 / 87.10$ \\
\hline CXCL-13 $(P<.001)$ & $0.832(0.737-0.904)$ & 0.677 at $<106.0$ & $76.74 / 90.91$ & $8.44 / 0.26$ & $89.19 / 80.00$ \\
\hline $\mathrm{IL}-10(P<.001)$ & $0.792(0.692-0.872)$ & 0.583 at $>21.77$ & $62.79 / 95.45$ & $13.81 / 0.39$ & $93.10 / 72.41$ \\
\hline $\begin{array}{l}\text { ADCavg, CXCL-13, IL-10 } \\
(P<.001)\end{array}$ & $0.928(0.851-0.972)$ & $\begin{array}{l}0.723 \text { at probability of } \\
>0.527\end{array}$ & $81.40 / 90.91$ & $11.26 / 0.25$ & $89.74 / 83.33$ \\
\hline
\end{tabular}

Note:-NPV indicates negative predictive value; PPV, positive predictive value.

${ }^{a}$ All ROC curves were statistically significant. Optimized thresholds were selected by using the maximum Youden Index J, the maximum vertical distance from the AUC $=0.5$ null hypothesis line (sensitivity [1-specificity]). PPV and NPV should be interpreted with caution because they are highly dependent on the prevalence of the disease in the tested population, and as CSF testing becomes more common, this may not be reflective of our study population.

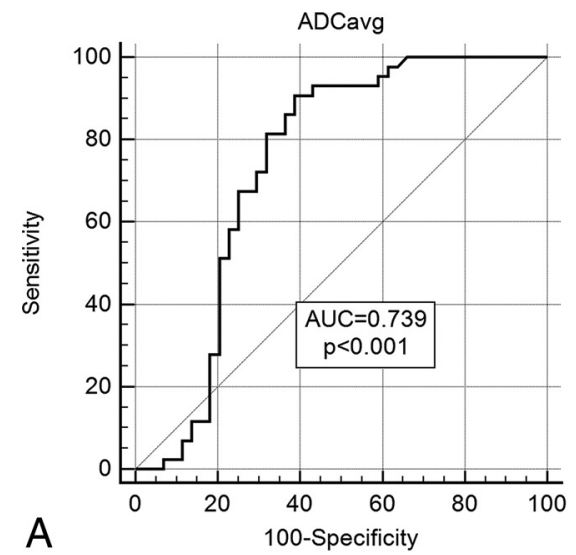

$\mathrm{IL}-10$

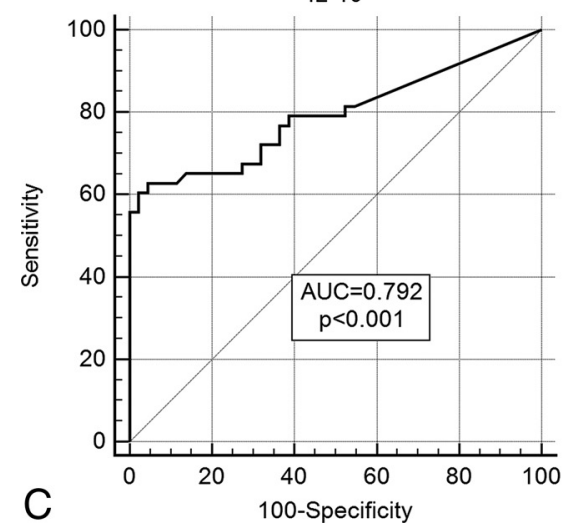

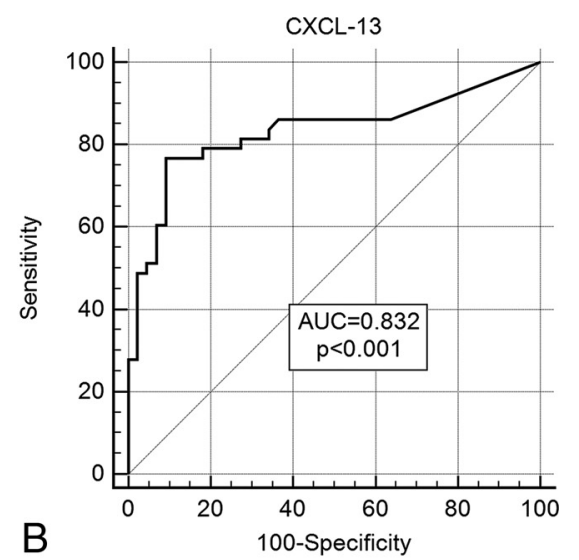

ADCavg, CXCL-13, and IL-10

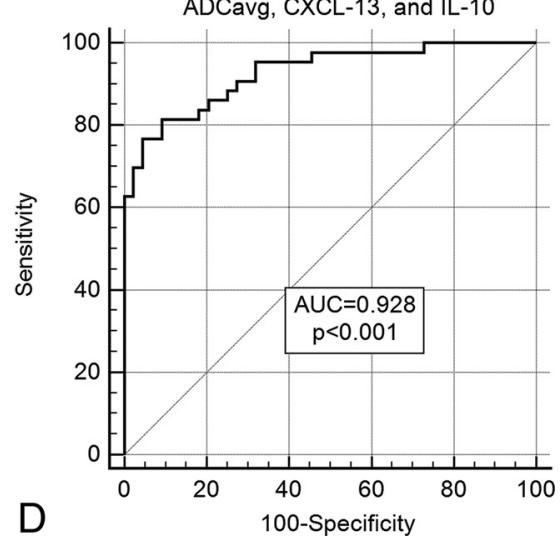

FIG 3. ROC curves of $A D C a v g(A), C X C L-13(B), I L-10(C)$, and $A D C a v g$ with $C X C L-13$ and IL-10 (D). All ROCs are statistically significant $(P<.001)$. Diagnostic performance measured by AUC is statistically significantly superior in the multiple-variable model $(D)$ compared with the single-variable models $(A-C)$.

retrospectively available for $64 / 87$ patients (30/43 patients with CNSL and 34/44 without it). More patients with CNSL were confirmed to have received corticosteroids $(n=24)$ before CSF sampling than those in the non-CNSL group ( $n=14, P=.005)$. The corticosteroid administered was dexamethasone in 22 and hydrocortisone in 2 of the patients with CNSL and dexamethasone in 11 , methylprednisolone in 2 , and prednisone in 1 of the patients without CNSL.

\section{ADCavg and CSF Values by Group}

ADCavg, CSF CXCL-13, and CSF IL-10 values are reported in Table 2 and depicted in Fig 2. ADCavg was statistically significantly lower and CSF CXCL-13 and IL-10 values were higher in CNSL than in non-CNSL $(P<.001)$.

\section{Single-Variable ROC for the Identification of CNSL}

ROC curves for all 3 variables analyzed were statistically significant for the identification of CNSL $(P<.001)$. Results with AUC and optimized thresholds with the maximum Youden Index J, sensitivities, specificities, likelihood ratios, and predictive values are reported in Table 3 and depicted in Fig 3. AUCs ranged from 0.739 (ADCavg) to 0.832 (CXCL-13), and maximum Youden Index J values ranged from 0.521 (ADCavg) to 0.677 (CXCL13). Threshold values for $95 \%$ specificity with associated sensitivity are reported in Table 4. Sensitivity at $95 \%$ specificity ranged from $0.00 \%$ (ADCavg, $\leq 598.2$ ) to $62.79 \%$ (IL-10> 20.65).

\section{Multiple Variable Logistic Regression for the Identification of CNSL}

The results of multiple-variable logistic regression are presented in Table 5. The model was overall statistically significant $(P<.001)$, and the 3 individual variables were each individually statistically significant. The logistic regression equation can be represented as: probability of CNSL $=1 /[1+$ $\left.\mathrm{e}^{(2.617-0.0048 \text { (ADCavg) }+0.0024 \text { (CXCL-13) +0.0626(IL-10) }}\right]$. The multiple-variable ROC results are presented in Table 3, and the curve is depicted in Fig 3. The AUC was 0.928, and the maximum Youden Index J was 0.723 , with sensitivity and specificity of $81.40 \%$ and $90.91 \%$ at a probability threshold of $>0.527$. Results at set $95 \%$ specificity are reported in Table 4 . Sensitivity at set $95 \%$ specificity was 76.74 at a probability threshold of $>.639$.

\section{ROC Comparisons}

Results of ROC curve comparisons are reported in Table 6. The multiple-variable ROC demonstrated statistically significantly better diagnostic performance than the single-variable AUCs.

\section{DISCUSSION}

While the diagnosis of CNSL can be suggested on the basis of MR imaging findings, arriving at a consistently accurate preoperative 
diagnosis for a patient with a brain mass lesion encountered on MR imaging remains an overall difficult task and an active area of research. ${ }^{9-13,18-20}$ Considerable advances, however, have been made in the field of CSF proteomics that have yielded 2 CSF biomarkers for CNSL, CXCL-13 and IL-10. ${ }^{22-25}$ In this study, we have examined the individual diagnostic performances of ADC, CSF CXCL-13, and IL-10 and the combined diagnostic performances in a multiple-variable model considering ADC, CSF CXCL-13, and CSF IL-10 in cohorts of patients with and without CNSL. We found that the combined use of ADC, CSF CXCL-13, and CSF IL-10 results in a statistically significantly increased diagnostic performance for the diagnosis of CNSL and that each variable has a statistically significant individual effect. CSF CXCL-13 and CSF IL-10 values should be considered when a brain mass with reduced ADC values is encountered and when the diagnosis of CNSL is considered. This study provides the statistical basis for considering all 3 variables in clinical practice. Consideration of these factors could potentially be used in the future to make the diagnosis of CNSL without the need for stereotactic biopsy.

Our study supports the observations in the literature that ADC values are decreased and CSF CXCL-13 and IL-10 values are increased in CNSL, while demonstrating the combined use of these variables in multiple-variable diagnostic models. ${ }^{9-13,18-26}$ ADCavg demonstrated a moderate diagnostic performance for CNSL in ROC analysis with an AUC of 0.739 (Fig 3) and was relatively sensitive compared with specificity, with an optimized sensitivity/specificity pair of 90.70/61.36 at a threshold of $\leq 971$. CSF CXCL-13 and CSF IL-10 also demonstrated moderate diagnostic performance as previously shown for CNSL in ROC analysis with AUCs of 0.832 for CXCL-13 and 0.792 for IL-10 (Fig 3). In agreement with our prior analysis and the literature, these CSF biomarkers (particularly IL-10) were found to be more specific than sensitive, with optimized sensitivity/specificity pairs of 76.74/90.91 for CXCL-13 (at > 106) and 62.79/95.45 for IL-10 (at $>21.77)^{22-25}$

Our multiple-variable model takes advantage of the different sensitivity/specificity profiles offered by each variable with result-

\begin{tabular}{|c|c|c|}
\hline Factor & $\begin{array}{c}\text { Sensitivity at Set } 95 \% \\
\text { Specificity }(95 \% \mathrm{CI})\end{array}$ & $\begin{array}{c}\text { Threshold Value for } \\
95 \% \text { Specificity }\end{array}$ \\
\hline ADCavg & $0.00(0.00-2.33)$ & $\leq 598.2$ \\
\hline CXCL-13 & 51.16 (18.60-79.07) & $>262.82$ \\
\hline IL-10 & 62.79 (48.84-79.07) & $>20.65$ \\
\hline $\begin{array}{l}\text { ADCavg, CXCL-13, } \\
\text { IL-10 }\end{array}$ & 72.09 (55.81-86.05) & Probability of $>.616$ \\
\hline
\end{tabular}

a ADCavg alone was essentially unable to reach a specificity of 95\% (the sensitivity at the calculated threshold is $0 \%$ ); however, with the addition of the CSF variables in the combined model, we can reach a specificity of $95 \%$ with a sensitivity of $72.09 \%$. The highest sensitivity at $95 \%$ specificity is reached in the multiple-variable model. ing improved and optimized diagnostic performance demonstrated by a statistically significantly larger AUC (0.928) on ROC analysis (Tables 3 and 6). The logistic regression results demonstrated that each of these variables had a statistically significant individual effect in the model and thus contributed to the probability of CNSL after the other variables had been taken into account. Our logistic regression equation can thus be used to calculate the probability of CNSL, given IL-10, CXCL-13, and ADCavg, and demonstrates that higher IL-10 and CXCL-13 values and lower ADC values should increase the diagnostic confidence for CNSL. Conversely, lower CXCL-13 and IL-10 values and higher ADC values should decrease diagnostic confidence for CNSL. These results statistically demonstrate the importance of considering CSF analysis in these patients and not relying solely on the presence of a mass with reduced diffusion in making the diagnosis of CNSL.

Our study contains several important limitations. The size of our study is relatively modest, and it was performed at only 1 institution. Clinical factors such as corticosteroid administration were not controlled, and more of the patients with CNSL received corticosteroids before CSF sampling than the patients without CNSL, the effect of which is unknown. A larger, prospective, multi-institutional study could be considered to further evaluate the results of this study. The quantitative measurement of ADC values as performed in this study may pose a limitation to adoption into clinical workflow; however, we routinely use a similar processing method on the same platform for perfusion analysis. Possible future directions to address this limitation could include evaluating the performance of subjectively "restricted diffusion" with CSF CXCL-13 and IL-10. Our multiple-variable models are limited by the variables that we included. With the CNSL group being older that the non-CNSL group, age could have been added to the model and likely would have had an effect; age was, however, omitted for simplicity in comparison of the ROC curves with the corresponding single-variable ROCs. Likewise, additional imaging features could have also been investigated but were omitted for model simplicity. Furthermore, the predictive model that we present is unlikely to be easily adopted in clinical practice; however, a version of the regression equation could conceivably be combined with automatic lesion segmentation to measure ADC and automated data extraction from the medical record for use in computer-aided diagnosis and decision support in the future. We likely have the computing power to accomplish this automated

Table 6: Comparisons of the multiple-variable ROC with the single-variable ROCs ${ }^{\mathrm{a}}$

\begin{tabular}{cccc}
\hline Model & ADCavg & CXCL-13 & IL-10 \\
\hline ADCavg, CXCL-13, IL-10 & $P<.001$ & $P=.016$ & $P=.002$ \\
\hline
\end{tabular}

${ }^{a}$ The multiple-variable ROC demonstrated a statistically significantly larger AUC than the corresponding single-variable AUCs, signifying statistically significantly superior diagnostic performance.

Table 5: Results of multiple-variable logistic regression with the ADCavg and CSF CXCL-13 and IL-10

\begin{tabular}{cccccc}
\hline Model & Overall $\boldsymbol{P}$ & Intercept & ADCavg Effect and (OR) & CXCL-13 Effect and (OR) & IL-10 Effect and (OR) \\
\hline ADCavg, CXCL-13, IL-10 & $<001$ & 2.617 & -0.0048 & 0.0024 & 0.0626 \\
& & & $P=.012$ & $P=.022$ & $P=.017$ \\
& & & $(0.995)$ & $(1.002)$ & $(1.065)$ \\
\hline
\end{tabular}

a The model was overall statistically significant, and all individual variables were individually statistically significant. This result demonstrates that all 3 variables should be factored into the diagnostic confidence that a lesion is a CNSL. 
analysis, though these features are not currently incorporated into clinical PACS workstations.

\section{CONCLUSIONS}

In this study, we have demonstrated that the combined use of ADC, CSF CXCL-13, and CSF IL-10 results in statistically significantly increased diagnostic performance for the diagnosis of CNSL compared with the diagnostic performance of ADC alone. Our multiple-variable diagnostic model demonstrated excellent diagnostic performance (AUC of 0.928 and optimized sensitivity/ specificity of 81.40/90.91), which was statistically significantly superior to the diagnostic performance of the individual variable models. Within this multiple-variable model, we found statistically significant individual effects for ADCavg (OR, 0.995; $P=$ .012), CXCL-13 (OR, 1.002; $P=.022$ ), and IL-10 (OR, 1.065; $P=$ .017 ) demonstrating that each variable contributed individually to the probability of CNSL. This study statistically demonstrates the importance of corroborating with CSF CXCL-13 and CSF IL-10 values (or suggesting they be obtained) when a brain mass with reduced $\mathrm{ADC}$ values is encountered and when the diagnosis of CNSL is considered. Higher CXCL-13 and IL-10 values and lower ADC values should all individually increase the diagnostic confidence that a lesion is a CNSL. A combined diagnostic model incorporating ADCavg, CSF CXL-13, and CSF IL-10 could potentially be used in the future to make the diagnosis of CNSL without the need for stereotactic biopsy.

Disclosures: Marc C. Mabray—RELATED: Grant: National Institutes of Health, Comments: Supported by National Institutes of Health T32 Grant while working on this project. Francisco E. Valles—RELATED: Grant: University of California, San Francisco Doris Duke Charitable Foundation Clinical Research Fellowship (Independent Cancer Research Foundation).

\section{REFERENCES}

1. Ferreri AJ. Risk of CNS dissemination in extranodal lymphomas. Lancet Oncol 2014;15:e159-169 CrossRef Medline

2. Hochberg FH, Baehring JM, Hochberg EP. Primary CNS lymphoma. Nat Clin Pract Neurol 2007;3:24-35 CrossRef Medline

3. van Besien K, Gisselbrecht C, Pfreundschuh M, et al. Secondary lymphomas of the central nervous system: risk, prophylaxis and treatment. Leuk Lymphoma 2008;49(suppl 1):52-58 CrossRef Medline

4. van der Sanden GA, Schouten LJ, van Dijck JA, et al; Working Group of Specialists in Neuro-Oncology in the Southern and Eastern Netherlands. Primary central nervous system lymphomas: incidence and survival in the Southern and Eastern Netherlands. Cancer 2002;94: 1548-56 CrossRef Medline

5. Besson C, Goubar A, Gabarre J, et al. Changes in AIDS-related lymphoma since the era of highly active antiretroviral therapy. Blood 2001;98:2339-44 CrossRef Medline

6. Haldorsen IS, Krossnes BK, Aarseth JH, et al. Increasing incidence and continued dismal outcome of primary central nervous system lymphoma in Norway 1989-2003: time trends in a 15-year national survey. Cancer 2007;110:1803-14 CrossRef Medline

7. Olson JE, Janney CA, Rao RD, et al. The continuing increase in the incidence of primary central nervous system non-Hodgkin lymphoma: a surveillance, epidemiology, and end results analysis. Cancer 2002;95:1504-10 CrossRef Medline

8. Coté TR, Manns A, Hardy CR, et al. Epidemiology of brain lymphoma among people with or without acquired immunodeficiency syndrome: AIDS/Cancer Study Group. J Natl Cancer Inst 1996;88: 675-79 CrossRef Medline

9. Cha S. Update on brain tumor imaging: from anatomy to physiology. AJNR Am J Neuroradiol 2006;27:475-87 Medline

10. Kickingereder P, Sahm F, Wiestler B, et al. Evaluation of microvascular permeability with dynamic contrast-enhanced MRI for the differentiation of primary CNS lymphoma and glioblastoma: radiologic-pathologic correlation. AJNR Am J Neuroradiol 2014;35: 1503-08 CrossRef Medline

11. Zacharia TT, Law M, Naidich TP, et al. Central nervous system lymphoma characterization by diffusion-weighted imaging and MR spectroscopy. J Neuroimaging 2008;18:411-17 CrossRef Medline

12. Haldorsen IS, Espeland A, Larsson EM. Central nervous system lymphoma: characteristic findings on traditional and advanced imaging. AJNR Am J Neuroradiol 2011;32:984-92 CrossRef Medline

13. Kickingereder $P$, Wiestler B, Sahm F, et al. Primary central nervous system lymphoma and atypical glioblastoma: multiparametric differentiation by using diffusion-, perfusion-, and susceptibility-weighted MR imaging. Radiology 2014;272:843-50 CrossRef Medline

14. Josephson SA, Papanastassiou AM, Berger MS, et al. The diagnostic utility of brain biopsy procedures in patients with rapidly deteriorating neurological conditions or dementia. J Neurosurg 2007;106: 72-75 CrossRef Medline

15. Hall WA. The safety and efficacy of stereotactic biopsy for intracranial lesions. Cancer 1998;82:1749-55 Medline

16. Weller M. Glucocorticoid treatment of primary CNS lymphoma. J Neurooncol 1999;43:237-39 CrossRef Medline

17. Geppert M, Ostertag CB, Seitz G, et al. Glucocorticoid therapy obscures the diagnosis of cerebral lymphoma. Acta Neuropathol 1990; 80:629-34 CrossRef Medline

18. Calli C, Kitis O, Yunten N, et al. Perfusion and diffusion MR imaging in enhancing malignant cerebral tumors. Eur J Radiol 2006;58:394403 CrossRef Medline

19. Horger M, Fenchel M, Nägele T, et al. Water diffusivity: comparison of primary CNS lymphoma and astrocytic tumor infiltrating the corpus callosum. AJR Am J Roentgenol 2009;193:1384-87 CrossRef Medline

20. Guo AC, Cummings TJ, Dash RC, et al. Lymphomas and high-grade astrocytomas: comparison of water diffusibility and histologic characteristics. Radiology 2002;224:177-83 CrossRef Medline

21. Barajas RF Jr, Rubenstein JL, Chang JS, et al. Diffusion-weighted MR imaging derived apparent diffusion coefficient is predictive of clinical outcome in primary central nervous system lymphoma. AJNR Am J Neuroradiol 2010;31:60-66 CrossRef Medline

22. Roy S, Josephson SA, Fridlyand J, et al. Protein biomarker identification in the CSF of patients with CNS lymphoma. J Clin Oncol 2008;26:96-105 CrossRef Medline

23. Sasagawa $Y$, Akai $T$, Tachibana $O$, et al. Diagnostic value of interleukin-10 in cerebrospinal fluid for diffuse large B-cell lymphoma of the central nervous system. J Neurooncol 2015;121:177-83 CrossRef Medline

24. Rubenstein JL, Wong VS, Kadoch C, et al. CXCL13 plus interleukin 10 is highly specific for the diagnosis of CNS lymphoma. Blood 2013; 121:4740-48 CrossRef Medline

25. Sasayama T, Nakamizo S, Nishihara M, et al. Cerebrospinal fluid interleukin-10 is a potentially useful biomarker in immunocompetent primary central nervous system lymphoma (PCNSL). Neuro Oncol 2012;14:368-80 CrossRef Medline

26. Valles FE, Perez-Valles CL, Regalado S, et al. Combined diffusion and perfusion MR imaging as biomarkers of prognosis in immunocompetent patients with primary central nervous system lymphoma. AJNR Am J Neuroradiol 2013;34:35-40 CrossRef Medline

27. DeLong ER, DeLong DM, Clarke-Pearson DL. Comparing the areas under two or more correlated receiver operating characteristic curves: a nonparametric approach. Biometrics 1988;44:837-45 CrossRef Medline 